九州大学学術情報リポジトリ

Kyushu University Institutional Repository

\title{
Barium-adularia from the Isagosawa Mine, Iwate Prefecture, Japan
}

Yoshimura, Toyofumi

Faculty of Sciences, Kyushu University

Shirozu, Haruo

Faculty of Sciences, Kyushu University

Kimura, Morihiro

Faculty of Sciences, Tokyo University

https://doi.org/10.5109/1524113

出版情報：九州大學理學部紀要：Series D, Geology. 4 (2), pp.163-166，1954-07-31. Faculty of Science, Kyushu University

バージョン：

権利関係 : 


\section{Barium-adularia from the Isagosawa Mine, Iwate Prefecture, Japan}

\section{By \\ Toyofumi YOSHIMURA, Haruo SHIROZU and Morihiro KIMURA}

White veinlets traversing through the hematite ore of the Isagosawa mine were found to be composed almost monomineralically of crystals of barium-adularia, which had been analysed by $\mathrm{H}$. SHIRORU with the following results (table 1).

Table 1. Chemical composition of barium-adularia

\begin{tabular}{lrrr} 
& wt. $\%$ & \multicolumn{2}{c}{ mol.\% } \\
$\mathrm{SiO}_{2}$ & 61.12 & $\mathrm{Or}$ & 88.6 \\
$\mathrm{Al}_{2} \mathrm{O}_{3}$ & 20.58 & $\mathrm{Cn}$ & 6.6 \\
$\mathrm{Fe}_{2} \mathrm{O}_{3}$ & 0.29 & $\mathrm{Ab}$ & 4.0 \\
$\mathrm{CaO}$ & 0.16 & $\mathrm{An}$ & 0.8 \\
$\mathrm{MgO}$ & none & & \\
$\mathrm{BaO}$ & 3.36 & & \\
$\mathrm{~K}_{2} \mathrm{O}$ & 13.87 & & \\
$\mathrm{Na}_{2} \mathrm{O}$ & 0.41 & & \\
$\mathrm{Igl}$ & 0.30 & & \\
& 100.12 & &
\end{tabular}

The crystalline aggregate of barium-adularia looks quite similar to calcite, showing distinct cleavage faces. It is not transparent, colour white with various shades of reddish brown.

Under the microscope it shows rhombic boundary, characteristic to adularia. Between the crossed nicols we can see mottled extinction of light and an abnormal interference colour, slight indigo-gray.

$$
\begin{aligned}
& \text { Specific gravity; } \quad \mathrm{d}_{4}^{11^{\circ}}=\mathbf{2 . 5 8 5} \\
& \text { Refractive indices ; } \quad \alpha=1.520-1.522 \\
& \gamma=1.526-1.528 \\
& (-) 2 \mathrm{~V}=75^{\circ}
\end{aligned}
$$

An X-ray powder photograph of the barium-adularia confirms its identification (text-fig. 1, no. 1 and table 2 , no. 1). It was made in $5.73 \mathrm{~cm}$ radius camera with $\mathrm{Cu}-\mathrm{K} \alpha$ radiation. A powder pattern of adularia from the Obira mine is compared in text-fig. 1, no. 2 and table 2, no. 2. A little difference between them suggests the smaller size of the crystal lattice of barium-adularia. The authors wish to thank 
Tab. 2. X-ray powder diffraction data for barium-adularia and adularia

\begin{tabular}{|c|c|c|c|c|c|c|c|}
\hline \multicolumn{2}{|c|}{$\begin{array}{l}\text { No. } 1 \\
\text { Barium-adularia } \\
\text { Isagnsawa mine }\end{array}$} & \multicolumn{2}{|c|}{$\begin{array}{l}\text { No. } 2 \\
\text { Adularia } \\
\text { Obira mine }\end{array}$} & \multicolumn{2}{|c|}{$\begin{array}{l}\text { No. } 1 \\
\text { Barium-adularia } \\
\text { Isagosawa mine } \\
(\text { cont } / d)\end{array}$} & \multicolumn{2}{|c|}{$\begin{array}{l}\text { No. } 2 \\
\text { Adularia } \\
\text { Obira mine } \\
\qquad\left(\text { cont }^{\prime} d\right)\end{array}$} \\
\hline I & $\mathrm{d}(\AA)$ & I & $\mathrm{d}(\AA)$ & I & $\mathrm{d}(\AA)$ & I & $\mathrm{d}(\AA)$ \\
\hline W & 6.60 & M & 6.63 & & - & VW & 1.428 \\
\hline \multirow[t]{2}{*}{ VW } & 5.90 & W & 5.94 & M & 1.409 & W & 1.407 \\
\hline & - & $\mathrm{VW}$ & 5.21 & W & 1.385 & W & 1.381 \\
\hline VW & 4.64 & VW & 4.64 & & - & W & (Q) 1.372 \\
\hline VS & 4.25 & VS & 4.29 & VW & 1.365 & & - - \\
\hline W & 3.95 & $\mathrm{M}$ & 3.97 & W & 1.340 & W & 1.340 \\
\hline VS & 3.78 & VS & 3.80 & & - & VW & 1.328 \\
\hline $\mathrm{S}$ & 3.49 & 5 & 3.50 & BW & 1.310 & W & 1.314 \\
\hline VS & 3.34 & VS & 3.36 & W & 1.289 & W & 1.289 \\
\hline VS & 3.25 & VS & 3.25 & W & 1.278 & W & 1.278 \\
\hline VW & 3.14 & VW & 3.11 & W & 1.261 & M & 1.258 \\
\hline $\mathrm{S}$ & 3.005 & $S$ & 3.005 & VW & 1.227 & VW & 1.228 \\
\hline $\mathrm{S}$ & 2.919 & $\mathrm{~S}$ & 2.919 & VW & 1.210 & VW & 1.217 \\
\hline $\mathrm{S}$ & 2.778 & $\mathrm{~S}$ & 2.786 & W & 1.197 & BW & 1.197 \\
\hline \multirow[t]{3}{*}{ BS } & 2.590 & BS & 2.597 & VW & 1.181 & $\mathrm{BW}$ & 1.182 \\
\hline & 一 & VW & 2.533 & VW & 1.158 & VW & 1.157 \\
\hline & - & VW & 2493 & W & 1.147 & W & 1.148 \\
\hline W & 2.421 ) & W & 2.421 ) & VW & 1.127 & W & 1.124 \\
\hline $\mathrm{W}$ & $2.390\}$ & W & $2.390\}$ & VW & 1.111 & VW & 1.109 \\
\hline \multirow[t]{2}{*}{ W } & 2.331 & W & 2.336 & VW & 1.099 & VW & 1.097 \\
\hline & $\overline{0}$ & $\mathrm{~W}$ & 2.291 & VW & 1.084 & W & 1.084 \\
\hline VW & 2.225 & VW & 2.231 & VW & 1.0540 & & - \\
\hline $\mathbf{S}$ & 2.169 & $\mathrm{~S}$ & 2.174 & & - & VW & 1.0348 \\
\hline $\mathrm{M}$ & 2.130 & $\mathrm{~s}$ & 2.135 & VW & 1.0268 & & - \\
\hline W & 2.065 & W & 2.070 & & - & VW & 1.0145 \\
\hline $\mathrm{M}$ & 2.017 & M & 2.017 & & 一 & VW & 0.9911 \\
\hline $\mathrm{M}$ & 1.975 & $\mathrm{M}$ & 1.979 & & - & VW & 0.9822 \\
\hline $\mathrm{M}$ & 1.928 & $\mathrm{~s}$ & 1.932 & VW & 0.9702 & VW & 0.9709 \\
\hline $\mathrm{M}$ & 1.861 & W & 1.865 & & 一 & VW & 0.9626 \\
\hline VW & 1.836 & & - & VW & 0.9455 & VW & 0.9444 \\
\hline VS & 1.802 & VS & 1.806 & & 一 & VW & 0.9291 \\
\hline VW & 1.776 & VW & 1.779 & & 一 & VW & 0.9174 \\
\hline VW & 1.751 & VW & 1.754 & & 一 & VW & 0.8986 \\
\hline \multirow[t]{2}{*}{ VW } & 1.723 & VW & 1.726 & VW & 0.8917 & VW & 0.8903 \\
\hline & - & VW & 1.703 & VW & 0.8828 & VW & 0.8807 \\
\hline \multirow[t]{2}{*}{ W } & 1.679 & W & 1.682 & & - & VW & 0.8740 \\
\hline & - & VW & 1.654 & VW & 0.8672 & BVW & 0.8668 \\
\hline W & 1.633 & $\mathrm{~W}$ & 1.633 & VW & 0.8548 & BVW & 0.8552 \\
\hline W & 1.594 & W & 1.594 & VW & 0.8412 & VW & 0.8415 \\
\hline W & 1.572 & W & 1.569 & VW & 0.8299 & W & 0.8305 \\
\hline W & 1.536 & M & 1.541 & & & & \\
\hline VW & 1.518 & VW & 1.515 & & & & \\
\hline $\mathrm{s}$ & 1.498 & $\mathrm{~S}$ & 1.500 & & & & \\
\hline VW & 1.478 & VW & 1.478 & & & & \\
\hline $\mathrm{BM}$ & 1.454 & BW & 1.452 & & & & \\
\hline BM & 1.430 & W & 1.436 & & & & \\
\hline \multicolumn{3}{|c|}{$\mathrm{Cu}-\mathrm{K} \alpha$ radiation } & Camera & & $r=5.7$ & & \\
\hline \multirow{6}{*}{$\begin{array}{l}\text { VS } \\
\text { S: } \\
\text { M: } \\
\text { W: } \\
\text { VW } \\
\text { B: } \\
\text { Q: }\end{array}$} & $\begin{array}{l}\text { very st } \\
\text { strong }\end{array}$ & & \}$_{\text {of }}^{\text {th }}$ & oath & & & \\
\hline & mediun & & & & & & \\
\hline & weak & & & & & & \\
\hline & very $w$ & & & & & & \\
\hline & broad & & & & & & \\
\hline & quartz & & & & & & \\
\hline
\end{tabular}


Mr. M. Miyahisa for providing the specimens of adularia from the Obira mine used in this work.

Text-fig. 1. X-ray powder diffraction photographs of barium-adularia and adularia.

No. 1. Barium-adularia, Isagosawa mine.

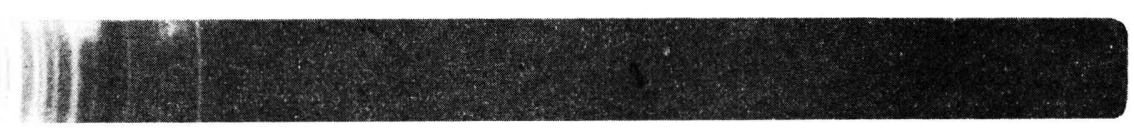

No. 2. Adularia, Obira mine.

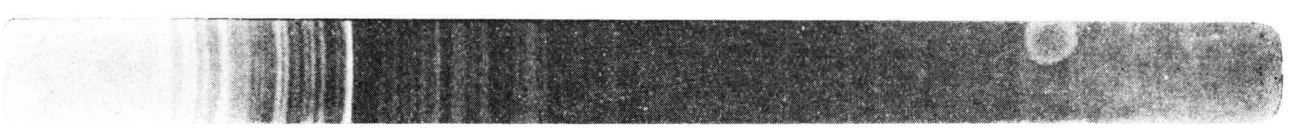

Barium-containing feldspars were reported in 1940 by T. YoshimurA* from the manganese deposits of the Kaso mine, Tochigi Pref. Japan. The iron ores of the Isagosawa mine do not contain much manganese, especially those of the higher ironcontent showing the less manganese. But they occur as lense-shaped metasomatic ore bodies in a diabasic dyke which traverses the strata of the Paleozoic formation, and seemingly related genetically to manganese deposits which are found in the Paleozoic region of this country.

* T. Yoshimura: Studies on the minerals from the manganese deposit of the Kaso mine, Japan; Jour. Fac. Sci. Hokkaido Imperial University, Ser. IV, Vol. IV, 313-451 (1939). 